\title{
Three Dimensional Ultrasonic Imaging
}

\section{Graham H. Thomas, Steve Benson, and Susan Crawford}

This paper was prepared for submittal to the SPIE OE/Aerospace Sensing

April 12-16, 1993

Orlando, FL

\section{March 1993}

This is a preprint of a paper intended for publication in a journal or proceed ings. Since changes may be made before publication, this preprint is made available with the understanding that it will not be cited or reproduced without the permission of the author. 


\section{DISCLAIMER}

This document was prepared as an account of work sponsored by an agency of the United States Government. Neither the United States Government nor the University of California nor any of their employees, makes any warranty, express or implied, or assumes any legal liability or responsibility for the accuracy, completeness, or usefulness of any information, apparat us, product, or process disdosed, or represents that its use would not infringe privately owned rights. Reference herein to any specific commercial products, process, or service by trade name, trademark, manufacturer, or otherwise, does not necessarily constitute or imply its endorsement, recommendation, or favoring by the United States Government or the University of California. The views and opinions of authors expressed herein do not necessarily state or rellect those of the United States Government or the University of Califomia, and shall not be used for advertising or product endorsement purposes. 


\title{
Three Dimensional Ultrasonic Imaging
}

\author{
Graham H. Thomas, Steve Benson, and Susan Crawford \\ Lawrence Livermore National Laboratory \\ PO Box 808, L-333 \\ Livermore California, 94550
}

\begin{abstract}
Ultrasonic nondestructive evaluation techniques interrogate components with high frequency acoustic energy. A transducer generates the acoustic energy and converts acoustic energy to electrical signals. The acoustic energy is reflected by abrupt changes in modulus and/or density which can be caused by a defect. Thus defects reflect the ultrasonic energy which is converted into electrical signals. Ultrasonic evaluation typically provides a two dimensional image of internal defects. These images are either planar views (C-scans) or crosssectional views (B-scans). The planar view is generated by raster scanning an ultrasonic transducer over the component and capturing the amplitude of internal reflections. Depth information is generally ignored. The cross-sectional view is generated by scanning the transducer along a single linf and capturing the amplitude and time of flight for each internal reflection. The amplitude and time of flight information is converted into an image of the cross section of the component where the scan was performed. By fusing the C-scan information with the B-scan information a three dimension image of the internal structure of the component can be produced. The three dimensional image can be manipulated by rotating and slicing to produce the optimal view of the internal structure.
\end{abstract}

The high frequency ultrasonic energy requires a liquid coupling media and thus applications for imaging in liquid environments are well suited to ultrasonic techniques. Examples of potential ultrasonic imaging applications are: inside liquid filled tanks, inside the human body, and underwater.

\section{Introduction}

Ultrasonic nondestructive evaluation is a powerful tool for finding defects and measuring material properties. In ultrasonics, high frequency acoustic energy is propagated into the component by a piezoelectric transducer and any abrupt acoustic impedance change will reflect part of the sonic energy. This reflected sound energy is sensed by a transducer and converted into an electrical pulse. The amplitude of the electrical signals are related to the defect size and the time of flight of the signal provides depth information. Raster scanning a focused ultrasonic transducer over the part generates a high resolution image of the reflector which provides information such as shape, depth and density of the reflector. Traditional ultrasonic imaging is based on either $X$ and $Y$ scanning, or 
$X$ scanning with $Z$ information provided by time of flight measurements. The reflector can be further accentuated by color coding the amplitude of the acoustic reflection from the defect. The $X$ and $Y$ scanning generates a plan view, and $X$ or $Y$ with depth scanning produces a cross sectional view. These two methods can be combined by capturing all the available information in the acoustic signal ard processing the data into a three dimensional image of the reflector. Three dimensional images are much easier to interpret since they display size shape location and orientation in a single picture. Additional image processing can provide slices, rotation, transparent surfaces, and enlargement to further aid defect characterization.

\section{Background}

Engineers, material scientists, welders, and others must make decisions based on the results of ultrasonic nondestructive evaluations. Displaying the ultrasonic results in a form that is complete and easily interpreted is important to these customers. Ultrasonic results are routinely displayed as planar (C-scans) and/or cross-sectional (B-scan) views. Planar views display size, shape, and location cf the reflector in the $x$ and $y$ plane. The cross-sectional view displays size, shape, and location of the reflector in the $x$ or $y$ and the $z$ plane. Combining the two views and implementing special processing algorithms allows the ultraso-ic data to be displayed in three dimensions. Once the results of the ultrasonic evaluation are in a three dimensional digital format, extensive software can be implemented which will rotate and slice the 3-D image to visualize the defect in any orientation. Thus the customer can quickly and easily see the defect and decisions regarding the component can be based on complete information about the flaw.

\section{Data Acquisition System}

Three dimensional images of ultrasonic evaluations depend on computer controlled scanning and digitized data acquisition. Each volume element in the three dimensional array represents an $X, Y$, and $Z$ coordinate and an amplitude measurement of the reflector. $X$ and $Y$ information is determined by scanner location. $Z$ information is calculated from the time of flight and the velocity of sound in the material. Amplitude or reflector strength is extracted from the digitized wave forms and stored as byte information. All of these calculations and measurements are performed by the computer.

An acoustic microscope (see Figure 1) was modified to capture full radio frequency $(R-F)$ wave forms. Most ultrasonic imaging is based on peak amplitude of the reflector. The acoustic microscope accurately scans the ultrasonic transducer over the component storing digital location information. A digitizing oscilloscope or an analog to digital converter then captures the full radio frequency wave form (see Figure 2) and stores it along with the location information. Time of flights and amplitudes are calculated from the stored R-F wave forms and combined with location data to generate a four dimensional matrix ( $X, Y, Z$, and amplitude). The time of flight determines the depth of the 
reflector and the amplitude is related to the strength of the reflector. From the information in the matrix, a three dimensional image is constructed on a workstation (see Figure 3).

Fast computers and large storage devices are necessary to generate and manipulate three dimensional ultrasonic images. For example there may be $1000 \mathrm{X}$ coordinates, $1000 \mathrm{Y}$ coordinates and $1000 \mathrm{Z}$ coordinates for a total of $10^{9}$ elements in the position matrix. This represents one gigabyte of information to be stored and processed. Data compression routines are desirable to efficiently handle the large amount of information and for archiving the data.

\section{Results}

Three dimensional ultrasonic imaging was demonstrated by scanning a test sample which was made by trapping porosity in an epoxy resin. The air bubbles serve as excellent ultrasonic reflectors and are distribute randomly through out the epoxy volume. Full ultrasonic wave forms were captured at each location in the raster scan, therefore $x, y$, and depth information was retained. This data was processed by a computer algorithm developed at Lawrence Livermore National Laboratory to construct a three dimensional image of the gas bubbles in the resin.

Tradition images were generated on the test sample to demonstrate the advantages of three dimensional ultrasonic imaging. . First the standard planar view called an ultrasonic C-scan, was generated as shown in Figure 4 . The planar view is a two dimensional picture of the bubbles with no depth information. Figure 5 is an ultrasonic B-scaii which displays a cross-sectional view along a single $x$ or $y$ line. The B-scan is also a two dimensional display. Finally, Figure 6 is a series of views which illustrate the power of visualizing the data in three dimensions. A video tape was made showing the three dimensional cube of ultrasonic data rotating. The motion aids the interpretation by displaying the motion of the defect revolving.

\section{Conclusions}

Three dimensional visualization of ultrasonic results is a method for representing large volumes of information in an easily interpreted manner. Unlike planar and cross sectional views, three dimensional images clearly display defect size, shape, location, and orientation in a single picture. This picture can be processed and manipulated to enhance the visualization of embedded defects For example the object can be rotated and/or sliced to see the defect at any angle.

To produce three dimensional images, large amounts of ultrasonic data must be captured and processed. Fortunately computer systems are improving rapidly. The CPU can process data at astounding speeds and the storage devices can hold extremely large amounts of data. For example a 1000 by 1000 raster scan with a 1000 point R-F wave form capture will generate a one gigabyte data set. 
The large amount of data to be stored and processed in three dimension ultrasonic imaging will benefit from advances in parallel processing and data compression. 


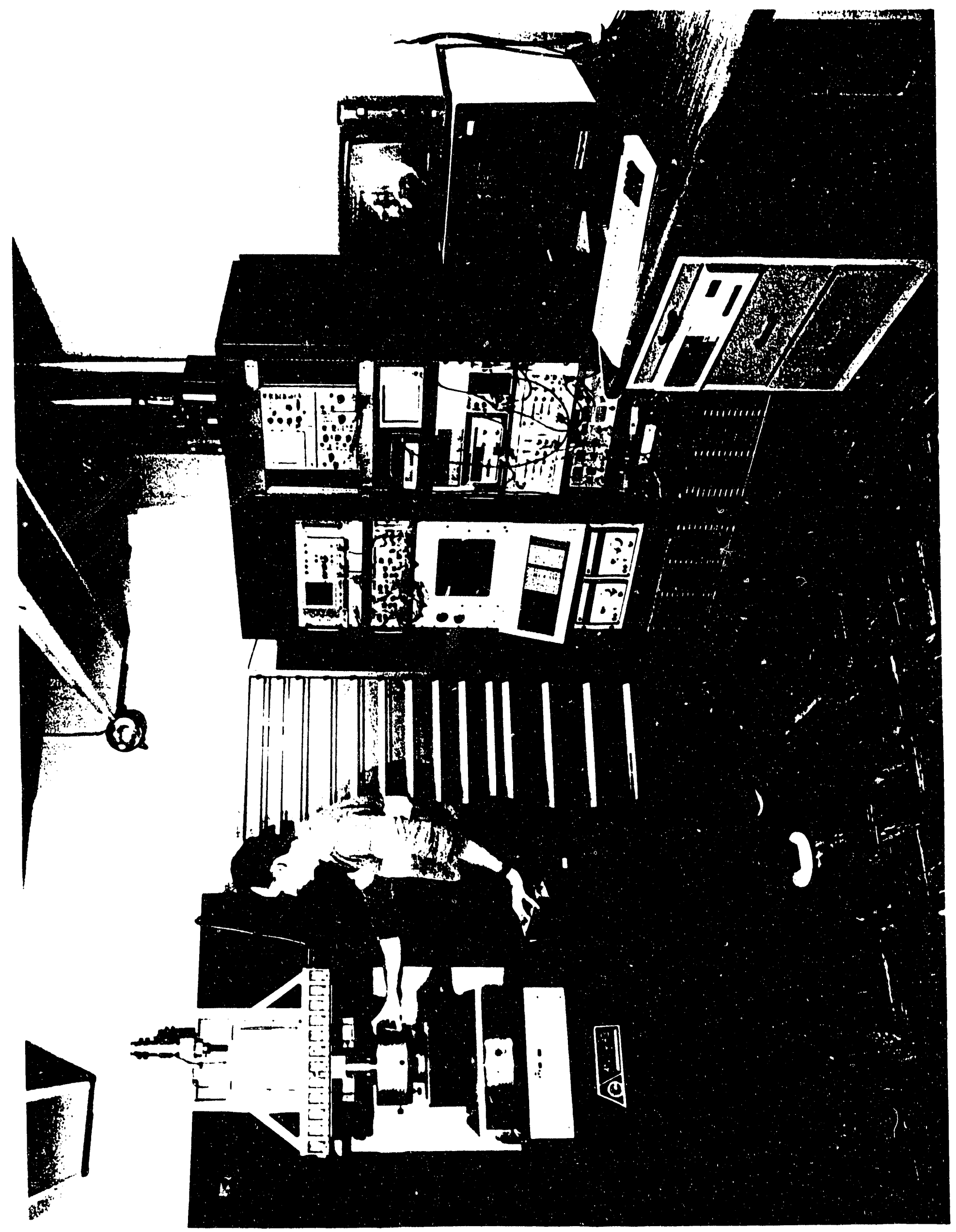

帒 


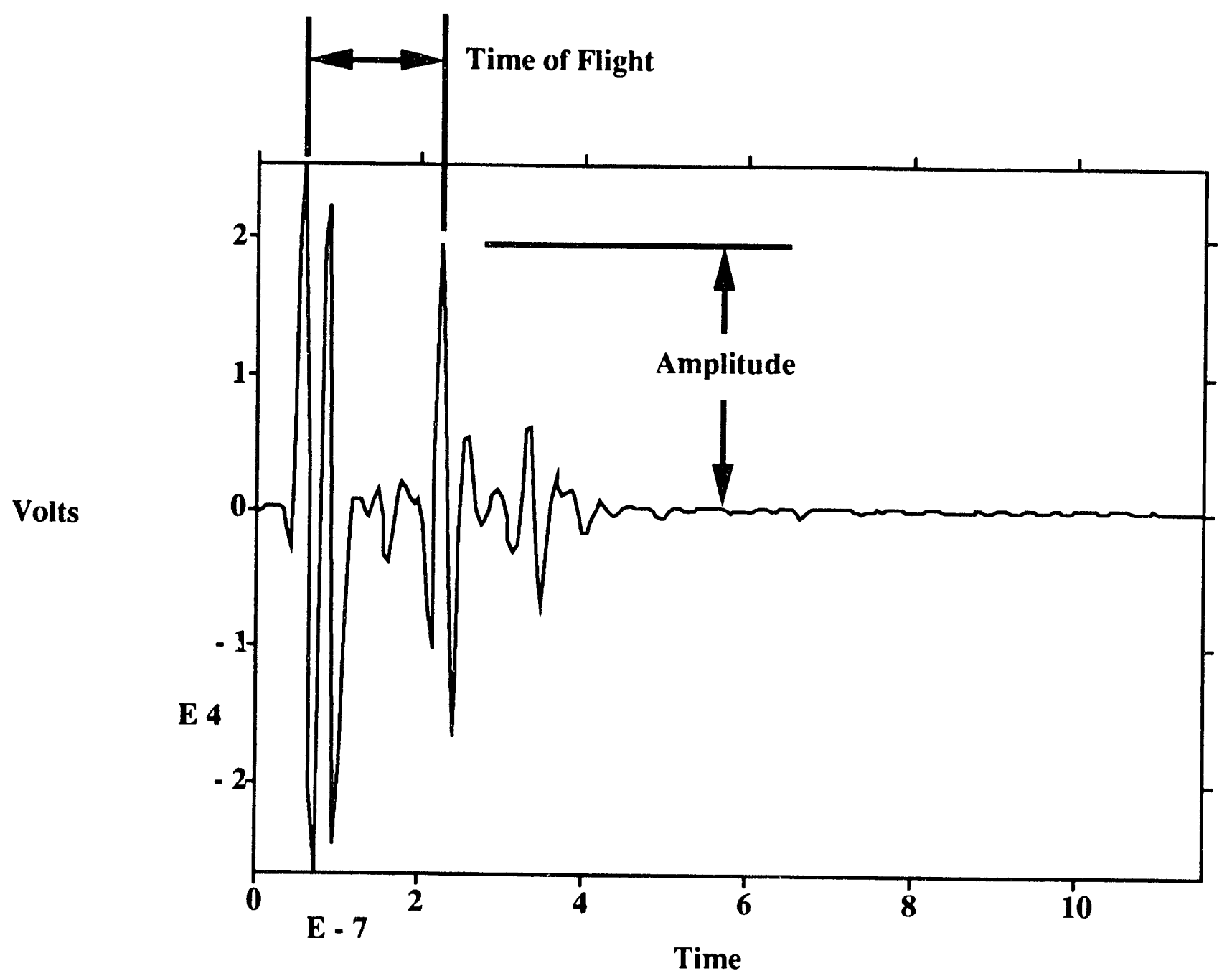

Figure 2 - Ultrasonic R-F wave formcaptured digitally. Note: time of flight and amplitude indications. 


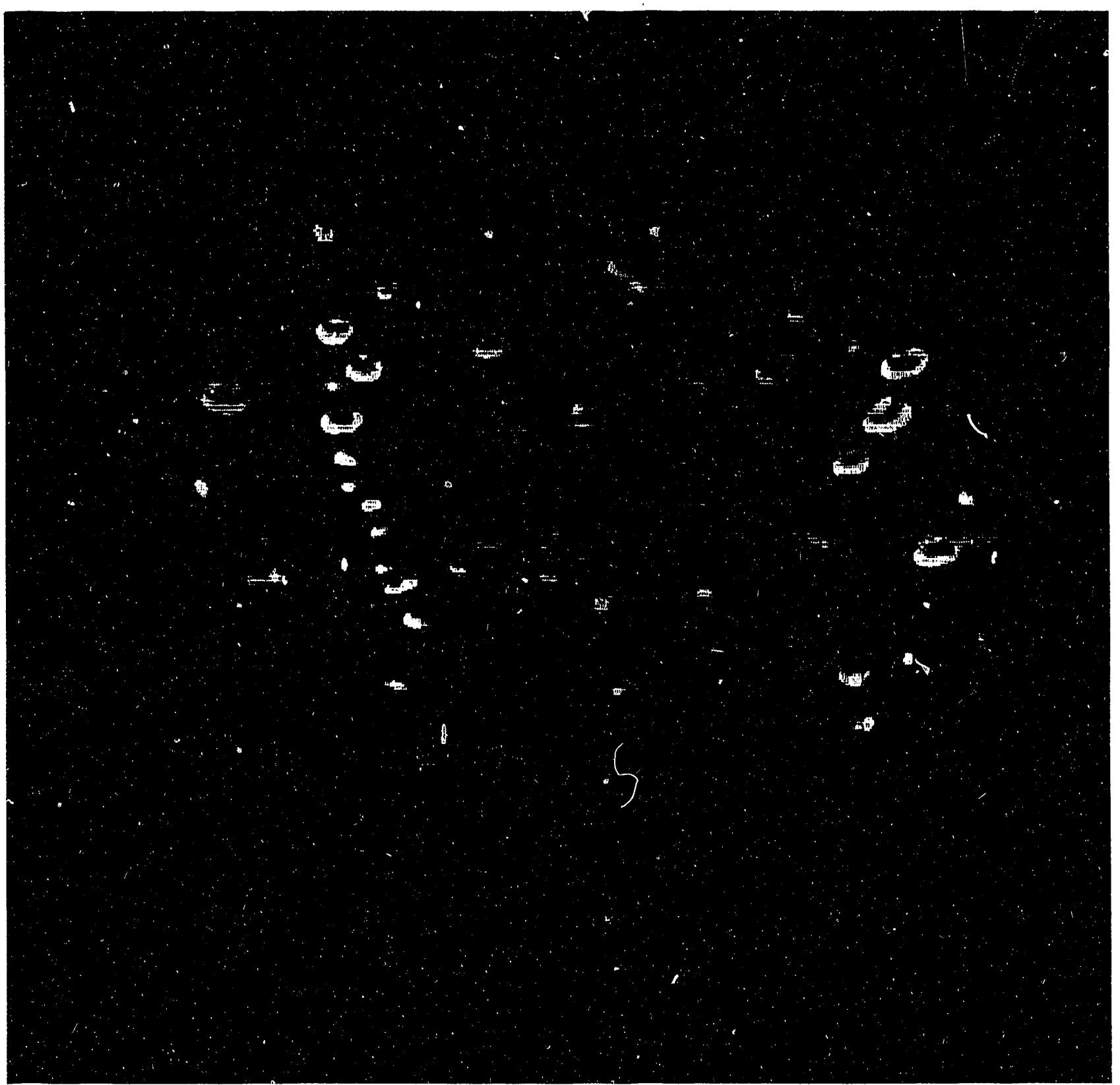

Figure 3 - Ultrasonic, three dimensional image of air bubbles trapped in epoxy 


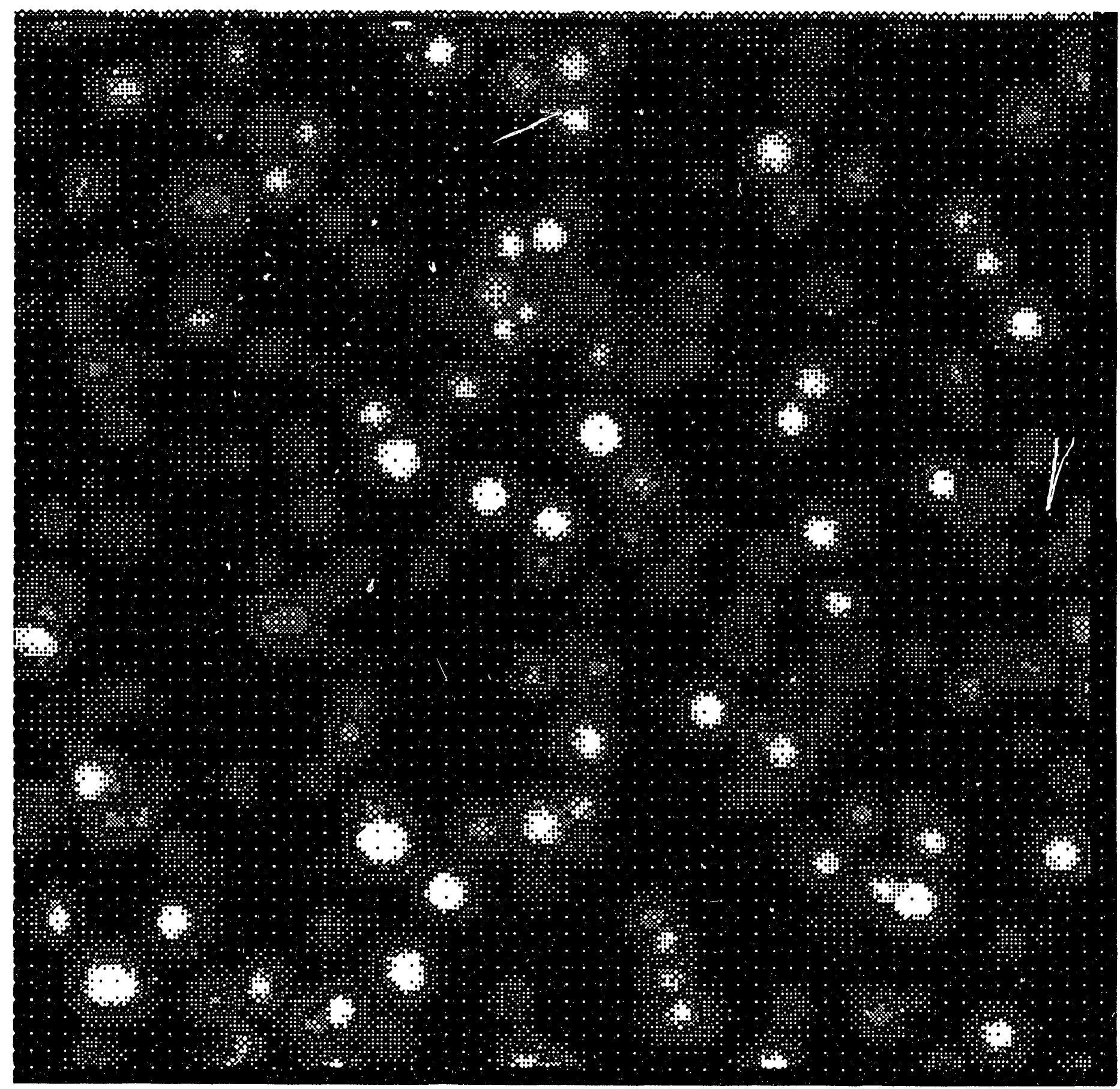

Figure 4 - Ultrasonic Planar view (C-scan) of air bubbles trapped in epoxy. No depth information is presented. 


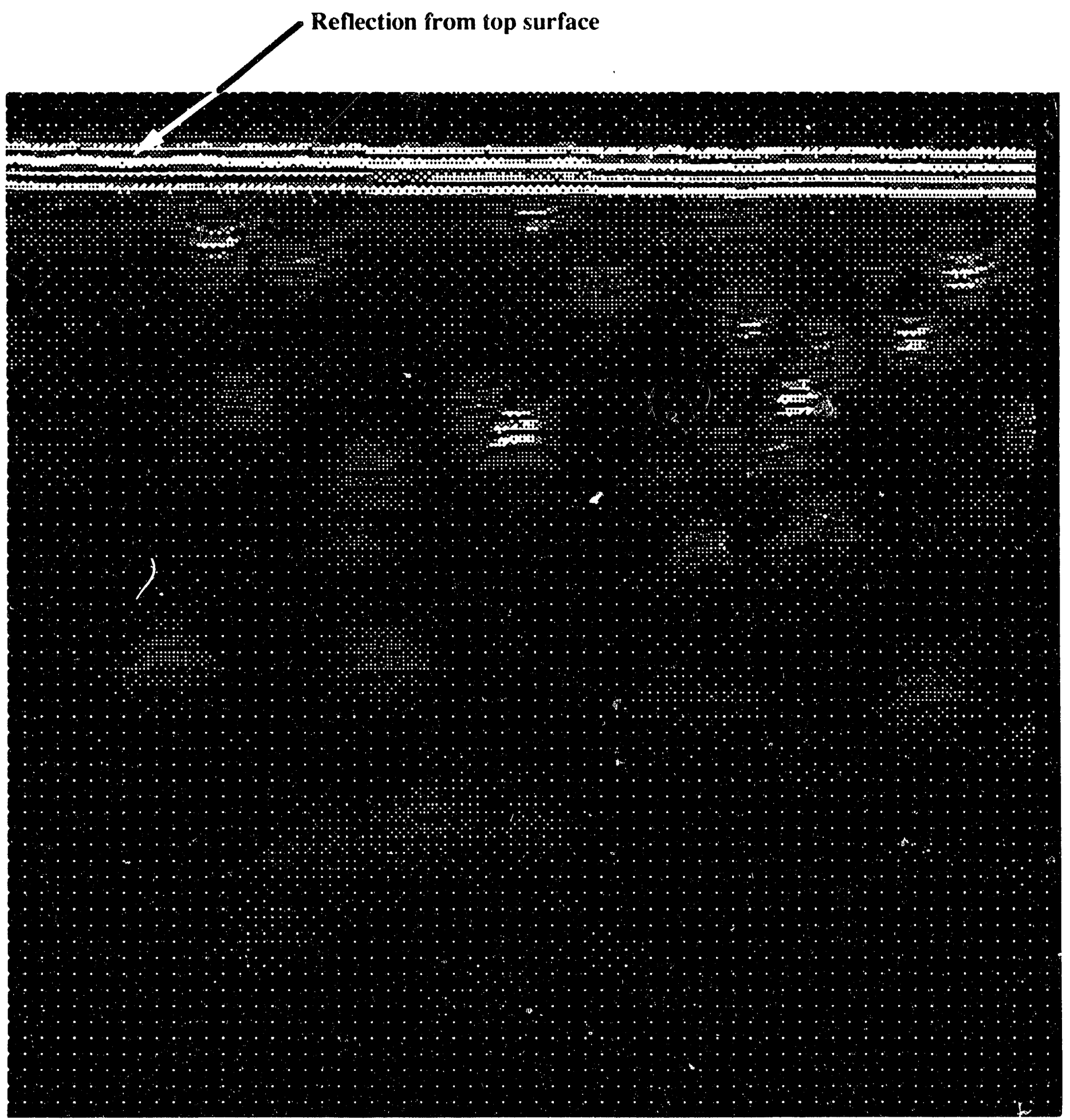

Figure 5 - Ultrasonic cross-sectional view (B-scan) along the X directionDisplays depth and X location information 


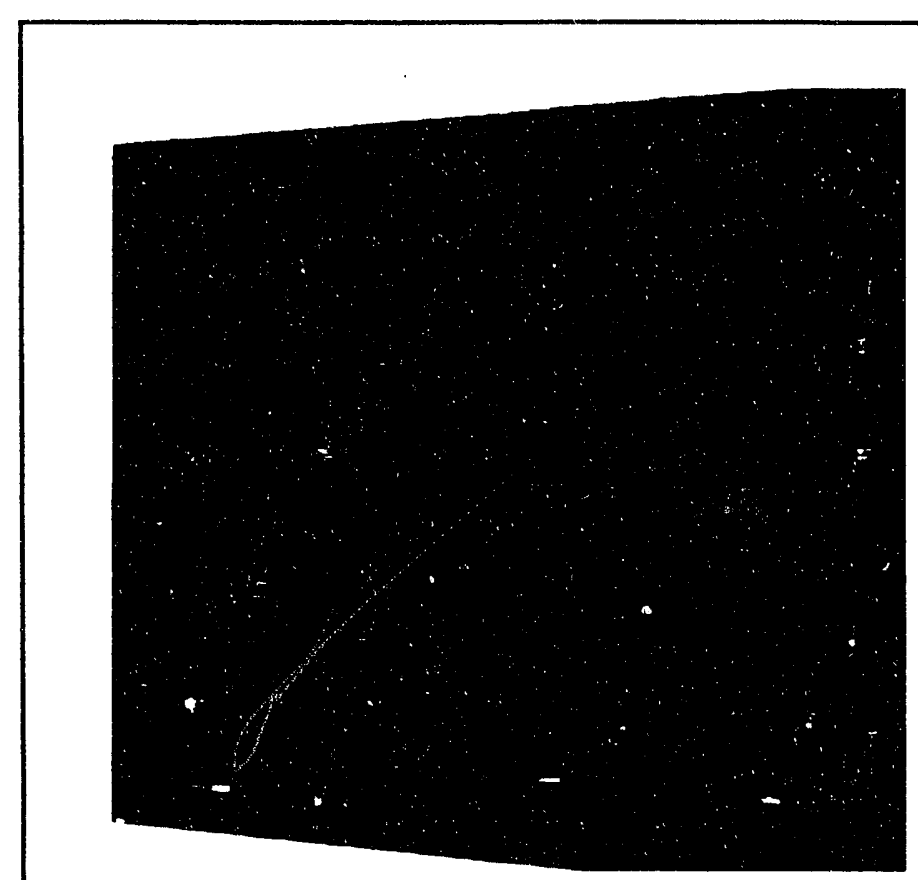

Figure 6a - 0 degree view

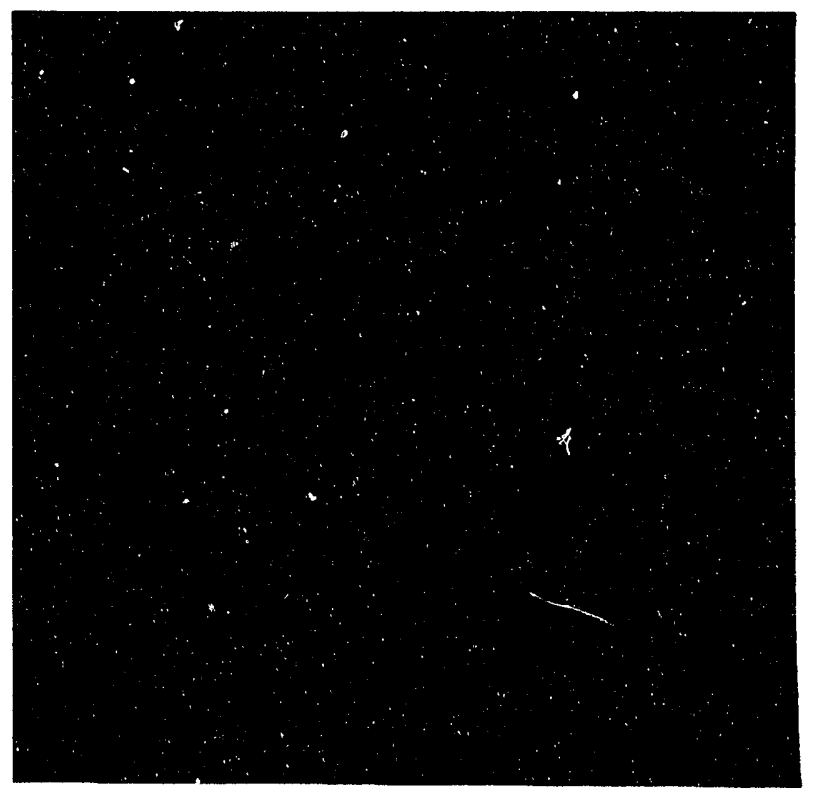

Figure 6c - Rotated 180 degrees

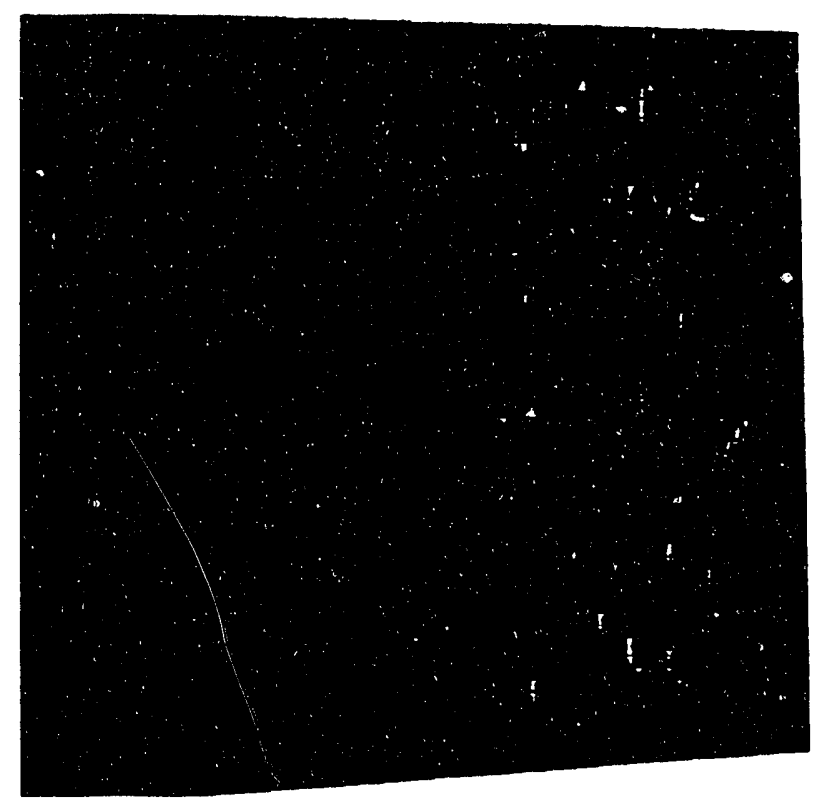

Figure 6b - Rotated 90 degrees

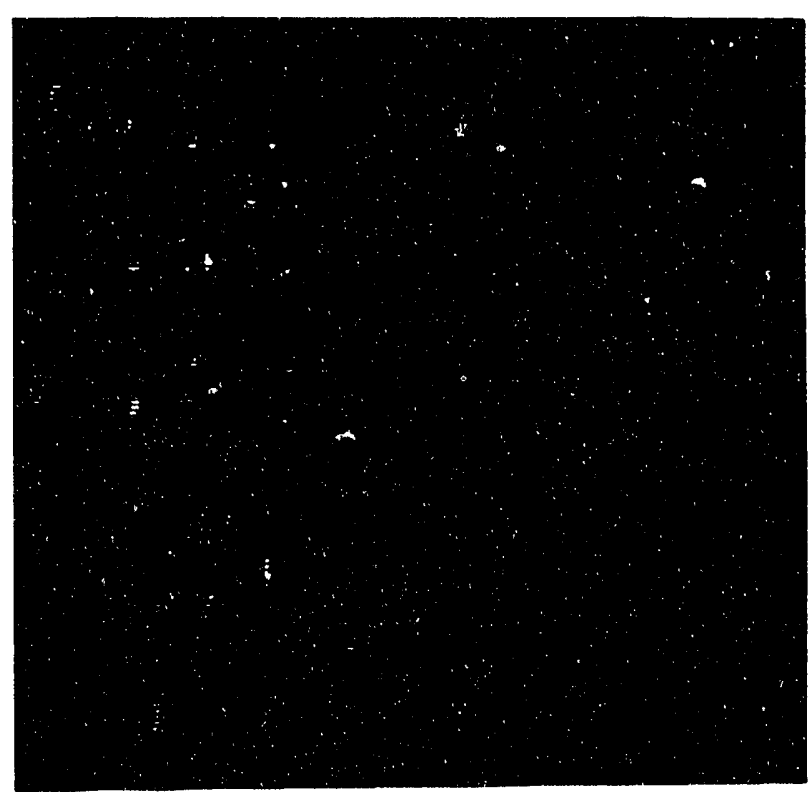

Figure 6d - Rotated 270 degrees

Figure 6 - Ultrasonic three dimensional displays of bubbles trapped in epoxy. Rotation of image presents information about size and location of reflectors. 

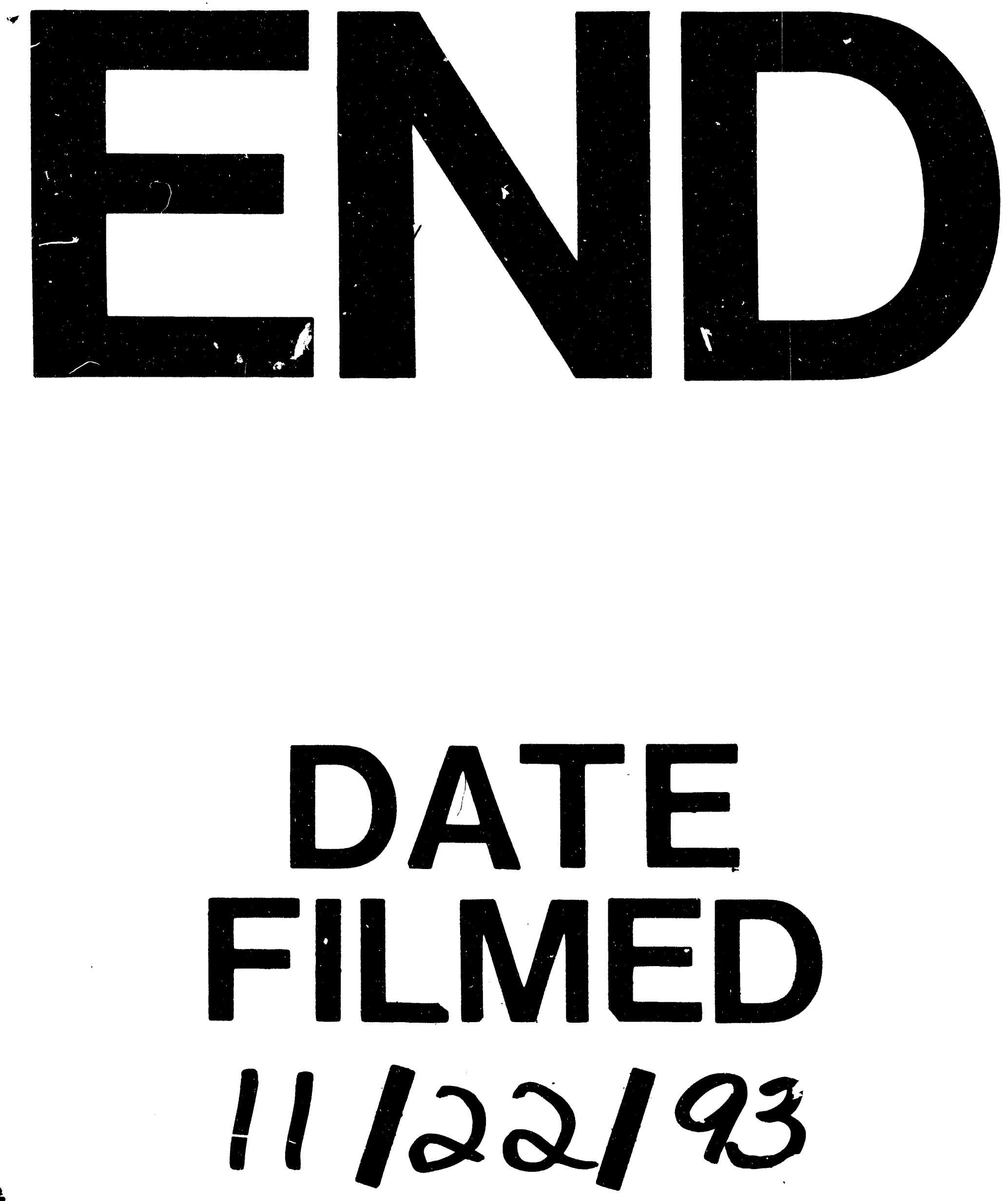

$\neq$ 
